\title{
CIBERMEDIOS LOCALES: EN LA ENCRUCIJADA POR LA INDEFINICIÓN DE MODELOS PARA TIEMPOS CONVULSOS
}

\author{
Xosé López García \\ (Universidad de Santiago de Compostela) \\ xose.lopez.garcia@usc.es
}

\begin{abstract}
Resumen: La crisis económica ha llevado a la mayoría de las editoras gallegas de diarios locales en papel a aplazar sus proyectos de renovación de las ediciones online. La fase de experimentación de la mano de la tecnología digital, que algunos medios pusieron en marcha a partir del año 2005, ha quedado hibernada. Ésta es una de las principales conclusiones de una investigación sobre la evolución de los cibermedios ${ }^{1}$ gallegos entre los años 2009-2010². El resultado de la radiografía de los cibermedios gallegos es una muestra de la tendencia general de los cibermedios locales en el momento actual ${ }^{3}$.
\end{abstract}

\footnotetext{
1 En este artículo emplearemos el término cibermedio para referirnos a los productos que permiten los procesos que van de un productor-emisor de contenidos y servicios informativos mediante estrategias y técnicas periodísticas específicas y adecuadas a las exigencias de la plataforma Internet y con la potencialidad incorporada por ésta, como el uso integrado de varios sistemas expresivos (escrito, gráfico, icónico, auditivo, audiovisual y multimedia), hasta llegar a unos usuarios que pueden seguir, manejar o producir otras informaciones y entablar diálogo o intercambiarse los papeles con el emisor. Esta es la definición que hace Mariano Cebrián en el número 33 de la revista Comunicar, titulado "Comunicación interactiva en los cibermedios". De la misma forma, emplearemos la denominación de ciberperiodismo para referirnos al periodismo que se hace en los cibermedios. Seguimos, por tanto, la definición de Ramón Salaverría, quien sostiene que el ciberperiodismo es la especialidad del periodismo que emplea el ciberespacio para investigar, producir $\mathrm{y}$, sobre todo, difundir contenidos periodísticos.
}

2 Este artículo recoge el resultado del proyecto de investigación "Tendencias en la convergencia dos medios de comunicación en Galicia 2007-2010” (PGIDT07PXIB212149PR), realizado por el Grupo Novos Medios. En el proyecto participaron los investigadores Xosé Pereira, Marita Otero, Berta García, Teresa de la Hera, Carlos Toural, Moisés Limia y María Dolores Calvo. El proyecto, conseguido en convocatoria pública, estuvo financiado por la Xunta de Galicia.

3. Los resultados del análisis del modelo gallego de cibermedios pueden extrapolarse al conjunto de los medios locales como una tendencia en el mapa de los cibermedios actuales. El motivo es la cohesión del modelo gallego, que ha servido para numerosas investigaciones como modelo de "estudio de caso" a causa de la red que conforman desde publicaciones comarcales hasta autonómicas. Los resultados de algunas de esas investigaciones del grupo Novos Medios están recogidos en los siguientes artículos: "La prensa popular gallega: un eslabón para la participación ciudadana", publicado en el número 3 de la revista ZER; "El modelo local de prensa gallega redefine estrategias para competir en la nueva era", publicado en el número 9 de la revista ZER; y "Modelos de ciberperiodismo gallego: de la definición a la consolidación de un nuevo entorno", publicado en el número 14 de la revista ZER. 

para tiempos convulsos

Palabras clave: ciberperiodismo, medios locales, contenidos locales.

Abstract:The economic crisis has led most of the Galician local newspapers' editors to postpone indepth renovation projects for their online editions. The testing phase brought by digital technology that some media launched in 2005 has been holed and most media make small adjustments to try to maintain their products with relative success. This is one of the main findings of a research project on the evolution of Galician online media between 2009 and 2010. The result of the Galician online media radiography is a sample of the general trend of local online media at the moment.

Keywords: cybermedia, journalism, local online media, local media, local journalism.

\section{INTRODUCCIÓN}

as empresas editoras de medios de comunicación locales impresos y online, como organizaciones que estructuran grupos de intereses, están, ciertamente, al servicio de los actores que las promueven, pero también tienen que crear marcos para garantizar los intereses de los profesionales -establecer ámbitos para el desarrollo de la práctica periodística- y precisan atender las necesidades y demandas de sus usuarios -para hacer realidad la utilidad pública de la información-. Y, al margen de la línea editorial de cada medio, para que funcione bien cualquier proyecto en el campo de la comunicación periodística, se precisa que todos los actores que intervienen en el proceso compartan un ámbito grande de coincidencias (intereses profesionales, empresariales y ciudadanos). Esos medios, como agentes de socialización, difunden un conjunto de ideas, valores y creencias que expresan intereses empresariales y profesionales (Borrat, 1997: 9-36). De todos los medios, durante mucho tiempo, el que situó como referente principal para la información reflexiva y de análisis fue el diario impreso en papel.

Estos principios generales, que presidieron buena parte de los análisis del funcionamiento de los medios de comunicación durante el siglo XX, siguen teniendo validez pero resultan insuficientes para explicar toda la complejidad del nuevo modelo y del nuevo escenario. Los propios medios de comunicación, en su transición a la era digital, constataron la necesidad de dar un salto cualitativo para intentar entender lo que está pasando. De manera especial, fueron los medios impresos los que, con una estrategia combinada para el papel y para el online, ensayaron a comienzos del tercer milenio fórmulas para nuevos modelos empresariales y nuevos modelos profesionales, sin el éxito que esperaban y con una oferta de pluralismo informativo que es más nominal que real (Reig, 2007: $10)$, en línea con el panorama de las últimas décadas del siglo $X X$.

\footnotetext{
${ }^{5}$ Durante los últimos años, varios premios Nobel de Economía han analizado las causas y efectos de la crisis económica de 2008. Para entender las consecuencias de esta crisis para los medios de comunicación me parecen especialmente clarificadoras las explicaciones de Joseph Stiglitz, premio Nobel de Economía en 2001. En una entrevista publicada por el diario español El País, en septiembre de 2008, ya avanzaba los principales peligros de la crisis que amenazaba los resultados de los medios de comunicación. http://www.elpais.com/articulo/primer/plano/crisis/Wall/Street/mercado/caida/muro/ Berlin/fue/comunismo/elpepueconeg/20080921elpneglse_11/Tes. Consultado: 22 de abril de 2011.)
} 
La crisis económica de finales de $2008^{4}$, que sacude a los países de nuestro entorno, puso en evidencia los puntos flacos de la estrategia que seguía la industria de los medios impresos, ya que la publicidad digital no reporta suficientes ingresos para compensar la pérdida en el campo del negocio tradicional $y$, además, el mayor impacto de su oferta informativa de la mano de las plataformas electrónicas no garantiza la prosperidad financiera. $Y$ es por eso que en los medios hay grandes recortes en las partidas destinadas a que los profesionales puedan hacer periodismo de calidad, el que es más útil a los ciudadanos para moverse en el mar de ruido y confusión informativa que reina en el escenario actual, y que la industria tradicional recorta en los programas de experimentación para encontrar nuevos caminos.

En definitiva, una encrucijada de la que, de momento, se vislumbra difícil salida.Ante un panorama como el descrito, los principales actores del sector redoblan los esfuerzos para construir vías de futuro que aseguren la continuidad de la industria y, al mismo tiempo, del periodismo como eje central de las sociedades actuales, ya que conforma una garantía para la existencia y funcionamiento de las sociedades democráticas, plurales y libres. Pero, ante las dificultades del actual contexto económico, optan por ajustes y por ralentizar planes innovadores que anhelan una mejor integración en el "mundo web" -especialmente en todo lo que se conoce como web2.05- y un mejor funcionamiento de sus productos en la red, sobre todo en el campo del negocio mediático a fin de rentabilizar las iniciativas.

\section{METODOLOGÍA}

El complejo panorama al que se enfrentan las editoras gallegas de medios locales impresos y online en esta fase de tránsito a la Sociedad de la Información y el Conocimiento, agravada por el actual panorama económico, se convirtió en una cuestión que concentró importantes debates en el ámbito mediático de Galicia. Esto animó al grupo Novos Medios 6 a incorporar estos aspectos en un proyecto de investigación sobre las tendencias de los medios de proximidad del "Finis Terrae" peninsular en relación con la convergencia periodística7, que es un proceso multidimensional facilitado por la implantación generalizada de las

\footnotetext{
${ }^{5}$ Para la definición de la web 2.0, seguimos el criterio establecido por Tim O'Reilly en el texto "Why is web 2.0". En línea, en la dirección http://oreilly.com/pub/a/oreilly/tim/news/2005/09/30/whatis-Web-20.html. Consultado: 14 de abril de 2011.)

${ }^{6}$ Novos Medios, grupo de investigación de la Universidad de Santiago (GI-1641), centra sus trabajos en el impacto de Internet en la evolución de las industrias culturales y en los procesos de comunicación -tanto mediáticos como generados por la autocomunicación-. ${ }^{7}$ Para hablar de convergencia se partió del documento de trabajo original e inédito elaborado para el proyecto "Convergencia digital en los medios de comunicación" (SEJ200614828-C06) por Ramón Salaverría, José Alberto García Avilés y Pere Masip en el año 2007. Algunos aspectos de esta definición, con pequeños cambios, se recogen en el libro Convergencia digital. Reconfiguración de los medios de comunicación en España (2010).
} 

para tiempos convulsos

tecnologías digitales (Salaverría, García Aviles. Masip, 2010: 59).

El estudio, además de las características de la convergencia en sus diferentes facetas y ámbitos -tecnológico, empresarial, profesional y editorial-8, quiso adentrarse en la reconfiguración del escenario mediático gallego, donde las empresas familiares son dominantes tanto en número como en su posición en el mercado9. El plan de trabajo buscaba analizar cómo la convergencia empresarial posibilitó la creación de alianzas, nuevos aprovechamientos del material informativo y renovados elementos formales de los contenidos.

El objetivo era, por tanto, disponer de un mapa de las características de los cibermedios gallegos puestos en marcha por las editoras de medios impresos diarios de pago, analizar su estrategia y sus proyectos $y$, finalmente, evaluar fortalezas y debilidades. De esta forma, se intentó conseguir una radiografía de un modelo de medios locales, como es el de los impresos y cibermedios de Galicia, a fin de conocer el origen de los problemas que encuentran los modelos de producto puestos en marcha, en todos los casos de acceso abierto y sin muro de pago.

Para conseguir el objetivo fijado, se aplicó el análisis de contenido10 durante tres meses -uno en cada año de la investigación, con dos muestras anuales de quince días, en marzo y octubre-, que permitió una primera aproximación al estado de la cuestión (el panorama y características de los cibermedios), que luego fue completada con entrevistas en profundidad y grupos de discusión. Los medios analizados fueron lavoz.es, farodevigo.es, elcorreogallego.es, elprogreso. es, laregion.es, elidealgallego.es y xornal.com, todos promovidos por empresas que editan diarios locales y multilocales. De la misma forma, para establecer elementos de comparación se analizaron dos medios "nativos digitales": vieiros. com (hoy desaparecido'11) y diariodegalicia.com.

El resultado de la investigación fue un completo dossier sobre la evolución

\footnotetext{
${ }^{5}$ Para el análisis de las diferentes facetas de la convergencia, la investigación también siguió el criterio establecido en el proyecto de investigación sobre la convergencia de los medios de comunicación en España entre los años 2006 y 2009 (SEJ2006-14828-C06). Los resultados de esta investigación pueden consultarse en el libro Convergencia digital. Reconfiguración de los medios de comunicación en España (2010).

${ }^{9}$ Los diarios controlados por OJD (La Voz de Galicia, Faro de Vigo, El Progreso y La Región) suman más de 160.000 ejemplares, mientras la prensa de Madrid de información general apenas supera los 50.000 ejemplares. Además, algo más de 20.000 ejemplares que computan para EI Mundo (prensa de Madrid) también hay que contarlos para El Correo Gallego (los dos se venden conjunta e inseparablemente pero, por acuerdo entre ambas cabeceras, el computo en OJD es para El Mundo).

10 El análisis de contenido se diseñó a partir de las aportaciones de Klaus Krippendorff, en su "Metodología del análisis de contenido: teoría y práctica", y de las consideraciones hechas en el diseño de varias investigaciones con análisis de contenido por parte del profesor José Luis Piñuel -en especial, sus aportaiones sobre la técnica del análisis de contenido-. Sus propuestas pueden consultarse en: http://web.jet.es/pinuel.raigada/index.html. Consultado: 14 de abril de 2011.

${ }^{11}$ Vieiros.com cerró el día 24 de julio de 2010, después de quince años en la red. Fue el primer medio "nativo digital" de información general en gallego.
} 
de los cibermedios gallegos en los años 2008, 2009 y 2010, con resultados del análisis del funcionamiento del modelo y de las características del producto.

\section{IMPACTO DE LA TECNOLOGÍA DIGITAL}

Después de cumplirse la primera década de la existencia de la prensa digital en España y Galicia (1995-2005)12, las empresas de comunicación mostraban en los contenidos y procesos productivos de todos sus medios -no sólo en los de Internet- las consecuencias directas e inmediatas del impacto de la tecnología digital. Se enfrentaban al reto de adoptar -y adaptar- nuevos modelos de gestión editorial multiplataforma que estaban contribuyendo a reconfigurar sustancialmente la oferta de contenidos, el perfil profesional de los periodistas y los procesos de gestión.

El impacto de la eclosión de la tecnología digital en los procesos editoriales, perfiles profesionales y modelos empresariales de los medios de comunicación de Galicia ha sido muy significativo. La creación de las nuevas infraestructuras para la comunicación digital favoreció la penetración de medios de otros ámbitos, por lo que se hizo más necesaria la producción de contenidos de proximidad para situarse con voz propia en el escenario mundial. Desde el primer momento todos los datos apuntaban a que estas nuevas infraestructuras de la fase de tránsito a la denominada Sociedad de la Información ${ }^{13}$, sin una respuesta de creación de contenidos de cercanías y de defensa de la diversidad regional en el ámbito mundial, son los "pipe-lines" de penetración unidireccional de contenidos externos y una vía potencial de erosión de las identidades locales (Díaz Nosty, 2007: 414). De ahí la importancia de las industrias locales de medios de comunicación y del papel de los medios gallegos, como modelo de medios de proximidad, en el escenario actual.

La denominada Sociedad de la Información, actualmente en fase de construcción, tiene su origen en la informatización de gran número de procesos y prácticas en el mundo industrial de servicios y con carácter general económico y social (Vidal Beneyto, 2002: 21). Y, por lo tanto, en las sociedades donde se desarrollen los nuevos valores de usos de las extensiones tecnológicas y se amplíe el tiempo de ocio y exposición a los medios, habrá unos resultados económicos mejores de los conocidos en los sistemas de medios menos evolucionados (Díaz Nosty, 2007: 414). Por lo tanto, semeja necesario activar tanto los planes de infraestructuras como los nuevos cometidos de los medios, así como de los usuarios, ahora con

\footnotetext{
${ }^{12}$ Elcorreogallego.es fue la primera versión en Internet de un diario local gallego en papel. El 12 de octubre de 1995 el Grupo Correo Gallego se convirtió en el primer medio de comunicación de Galicia en disponer de edición en Internet: elcorreogallego.es. ${ }^{13} \mathrm{De}$ las diferentes convenciones para denominar la sociedad actual, elegiremos para este trabajo la de Sociedad de la Información por ser la denominación que le ha dado Unesco en la cumbre mundial celebrada en el año 2003 en Ginebra y en el año 2005 en Túnez.
} 

para tiempos convulsos

mayor capacidad de intervención en los procesos.

La importancia de la industria de la comunicación de un país resulta, pues, estratégica en el siglo XXI, en esta fase de grandes cambios en las redes y con nuevos cometidos para los propios medios de comunicación, que ahora tienen novedosos marcos de relaciones con los usuarios. En el caso de Galicia, se trata de una industria con una larga tradición, siempre muy vinculada a la cercanía y con diferentes grados de compromiso con los intereses del país a lo largo de las sucesivas etapas históricas. En todo caso, se trata de un sector industrial importante por el volumen de negocio y cantidad de empleos que crea, pero sobre todo por su cometido de informar a los ciudadanos sobre lo que acontece en el país y en el mundo. Tiene un papel central en la conformación de la opinión pública gallega y, además, ahora es vital su concurso para afrontar los nuevos retos en la Sociedad de la Información.

La industria de los medios de comunicación en Galicia se asentó desde finales del siglo XIX y comienzos del siglo XX alrededor de los medios impresos, con la posterior incorporación de la radio (en el año 2008 se cumplieron 75 años de su venida a Galicia) y de la televisión (ya van más de tres décadas de la llegada de la televisión, pero en el campo industrial hay que referirse especialmente la etapa posterior al nacimiento de la Televisión de Galicia, que empezó sus emisiones el 25 de julio de 1985). Por tanto, a comienzos del siglo XXI, esta industria mantiene como pilares básicos los medios impresos, los audiovisuales e Internet. Tenemos, pues, tres ejes básicos sobre los que descansa un panorama de medios muy fragmentado y con un mercado relativamente pequeño ${ }^{14}$.

En el campo de la prensa y de las editoras locales de diarios de pago, tema que nos interesa en este caso, la propiedad familiar y la debilidad empresarial son dos de sus características que la definieron hasta la fase de tránsito al tercero milenio. Los resultados oficiales que presentaban las empresas de prensa en los últimos años indicaban beneficios en su cuenta de explotación y mejoras tanto en la difusión como en los ingresos publicitarios. Los datos preocupantes radicaban, especialmente, en su debilidad empresarial, que se manifestaba en la poca capacidad propia de capitalización y de innovación, en la escasa atención a la formación, en la inestabilidad en los equipos de gestión y en la excesiva dependencia de las ayudas oficiales (sobre todo gubernamentales). Otro punto débil residía en la línea informativa, poco equidistante de los distintos actores que intervenían en la sociedad gallega, especialmente de los poderes públicos y de los grandes grupos económicos (Campos y López, 2000: 24).

\footnotetext{
${ }^{14} \mathrm{Si}$ nos fijamos en la situación de la prensa en Galicia, vemos como un $36,7 \%$ de la población mayor de 14 años lee prensa a diario, pero un $26,8 \%$ no lee nunca los periódicos. En el caso de la radio, los datos nos dicen que un $42 \%$ de la población mayor de 14 años escucha la radio cada día, mientras que también es elevado el porcentaje que no la escucha nunca, llegando a un 29,8\%. En lo referente a la televisión, apreciamos como el número de horas diarias dedicadas a ver televisión entre la población mayor de 14 años se sitúa entre una hora y dos, con un 42,3\%. Fuente: Instituto Gallego de Estadística.
} 
En este contexto nacieron y se desarrollaron los cibermedios gallegos, promovidos por las editoras de medios impresos de información general de pago. Desde El Correo Gallego, el más madrugador en situarse en la red de redes, hasta La Voz de Galicia15, uno de los últimos en hacerlo, apostaron por la galaxia Internet, con estrategias orientadas a no quedar al margen de los cambios que se anunciaban en el sector y a pequeñas dosis de experimentación, a partir de los modelos más convencionales existentes en ese momento.

\section{LOS CAMBIOS AL NUEVO SOPORTE}

En el año 2010, todos los diarios en papel de información general de pago contaban con versión para la web. El desarrollo del periodismo en Internet, el ciberperiodismo, supuso un giro importante en el panorama mediático, al tiempo que el nuevo producto introducía elementos novedosos, como la confluencia del papel del emisor y del receptor y la facilidad para publicar contenidos en la red. Los diarios impresos de información general de pago entraron progresivamente en la esfera digital, pero tardaron varios años en advertir que no se trataba de una simple cuestión de traslación de textos a un nuevo soporte. Durante el primer lustro de vida de los cibermedios, casi no hubo cambios en la denominación y número de secciones de los digitales con respecto a la versiones en papel (Armentia et al., 2000: 29), hecho que confirmaron los cibermedios promovidos desde Galicia.

En esa época, Internet también avanzaba en nuestra comunidad autónoma, especialmente desde el año 2005. En el año 2007, algo más de un tercio de los gallegos (el $34 \%$ ) de entre 16 y 74 años emplearon la red de redes todas las semanas de octubre a diciembre y cuatro de cada diez (el $43 \%$ ) accedieron a ella por lo menos en una ocasión a lo largo de esos tres meses ${ }^{16 .}$. El dato de un tercio de los gallegos empleando la red todas las semanas suponía, por primera vez, una base real para el funcionamiento de los cibermedios con un público potencial relativamente importante. A partir de ese momento, el índice de usuarios de Internet en Galicia siguió creciendo, superando el $50 \%$ empleando la red de redes toda la semana, hasta las cifras actuales, que sitúan el porcentaje en el $69,7 \%$ de gallegos que, a finales de 2010 , emplearon la red todas las semanas de octubre a diciembre17.

A partir del año 2005, los cibermedios gallegos también empezaron a abando-

\footnotetext{
${ }^{15} \mathrm{La}$ Voz de Galicia, el diario de mayor difusión en Galicia, con más de cien mil ejemplares, según OJD, puso en marcha su edición digital en el año 2000, el 17 de mayoEstadística.).

${ }^{16} \mathrm{El}$ dato corresponde a la Encuesta sobre el equipamiento y el empleo de tecnologías de la información y la comunicación en los hogares en el año 2007. En línea, en http://www.ine.es. Consultado: 1 de abril de 2011.

${ }^{17}$ El dato corresponde a la misma Encuesta sobre el equipamiento y el empleo de tecnologías de la información y la comunicación en los hogares en el año 2010. En línea, en http://www.ine.es (http:// www.ine.es/jaxi/tabla.doc). Consultado: 1 de abril de 2011.
} 

para tiempos convulsos

nar algunos parecidos con las ediciones en papel de las empresas promotoras y diseñaron productos más pensados para la red. A medida que las herramientas facilitaban que los usuarios difundiesen sus contenidos, los cibermedios gallegos también abrían ventanas a la participación, aunque sin proyectos que arriesgasen por modelos renovadores. Y fue lavoz.es el primer cibermedio que el 5 de marzo de 2003 comenzó a publicar "viaje a la frontera", de David Beriain, enviado especial a Iraq con motivo de la Segunda Guerra del Golfo. Era el despegue de la primera bitácora que tenía cabida en un periódico español. Y en el año 2006, elcorreogallego.es inauguró una sección titulada "Periodismo cidadán" (Calvo, López, Otero, 2009: 54).

Fueron las dos primeras iniciativas participativas, que luego tuvieron continuidad con nuevas propuestas para que los ciudadanos enviasen sus aportaciones e interviniesen en la conversación, pero manteniendo la práctica profesional como el eje conductor en la elaboración de la práctica totalidad de los mensajes periodísticos. Desde ese momento, los cibermedios gallegos promovidos por las empresas editoras de diarios también prestaron renovada atención a la construcción de piezas hipertextuales y multimedia, así como a mejoras en la actualización del producto. La mayoría de esos cibermedios tenían una personalidad más pensada para la red y se lanzaban a la experimentación.

\section{DEBILIDADES DE LOS MEDIOS}

La fase de experimentación, que se desarrolló sobre todo en los años 2007 y 2008, estuvo caracterizada por un gran número de iniciativas, por muchas debilidades y, desde comienzos del año 2009, marcadas por la sombra de la duda a causa de la crisis económica. Las conclusiones de su estudio, que llevamos a cabo en el año 2008, ya apuntaban a que los cibermedios gallegos no aprovechaban las potencialidades del soporte telemático para ofrecer un producto novedoso, la frecuencia de actualización era baja, la contextualización estaba poco trabajada y había una escasez de elementos hipertextuales, multimedia e interactivos, lo que limitaba las posibilidades de un ejercicio periodístico participativo y dialogante (Calvo, López, Otero, 2009: 114).

Los datos de la investigación del año 2009 y 2010 ponen sobre la mesa muchas debilidades de los cibermedios gallegos, sobre todo en lo referido a su adaptación a las características del nuevo medio. Uno de los datos más sor-

\footnotetext{
${ }^{17} \mathrm{El}$ dato corresponde a la misma Encuesta sobre el equipamiento y el empleo de tecnologías de la información y la comunicación en los hogares en el año 2010. En línea, en http://www.ine.es (http://www.ine.es/jaxi/tabla.doc). Consultado: 1 de abril de 2011.
} 
prendentes es la baja actualización de la mayoría de las cabeceras, que sólo es de una vez al día. La excepción la constituyen lavoz.es y elcorreogallego.es, cibermedios que mantienen una actualización constante (varias veces al día, de acuerdo con las necesidades que marca la actualidad).

\begin{tabular}{|c|c|}
\hline Categoria & Publicaciones \\
\hline Bajo & La Región, El Progreso, El Ideal Gallego \\
\hline Medio & Faro de Vigo, Xornal de Galicia \\
\hline Alto & La Voz de Galicia, El Correo Gallego \\
\hline
\end{tabular}

Grado de actualización. Fuente: elaboración propia.

De la misma forma, se constata poca experimentación y planteamientos conservadores a la hora de arriesgar tanto en la construcción hipertextual como multimedia. El bajo número de enlaces internos, que se recoge en el siguiente gráfico, refleja aspectos del bajo índice de desarrollo e innovación de la mayoría de los cibermedios de proximidad de Galicia.

\begin{tabular}{|l|c|c|c|c|}
\cline { 2 - 5 } \multicolumn{1}{c|}{} & \multicolumn{4}{c|}{ Destino } \\
\cline { 2 - 5 } \multicolumn{1}{c|}{} & Extemo & Interno & Anbos & Totales \\
\hline El Correo Gallego & 4 & 167 & & 167 \\
\hline Faro de Vqo & 1 & 77 & & 78 \\
\hline El Ideal Gallego & & & & \\
\hline El Progreso & 9 & 36 & 2 & 47 \\
\hline La Región & & 58 & & 58 \\
\hline La woz de Galioja & 7 & 506 & 4 & 517 \\
\hline Xomal & & 128 & 123 & 251 \\
\hline Totales & 21 & 958 & 129 & 1118 \\
\hline
\end{tabular}

Fuente: Elaboración propia.

De los datos generales de los años 2009 y 2010 se deduce una progresiva desaparición de los textos de las noticias de portada o la reducción del texto noticioso a los titulares como elemento informativo predominante. La información, por lo tanto, queda resumida primordialmente en titulares de dos líneas. Por su parte, los elementos visuales ocupan un espacio relevante. Las fotografías, de tamaño mediano y pequeño, por este orden, aparecen con frecuencia, mientras a continuación se sitúan los recursos interactivos, representados con diferencia 
por la inserción de comentarios.

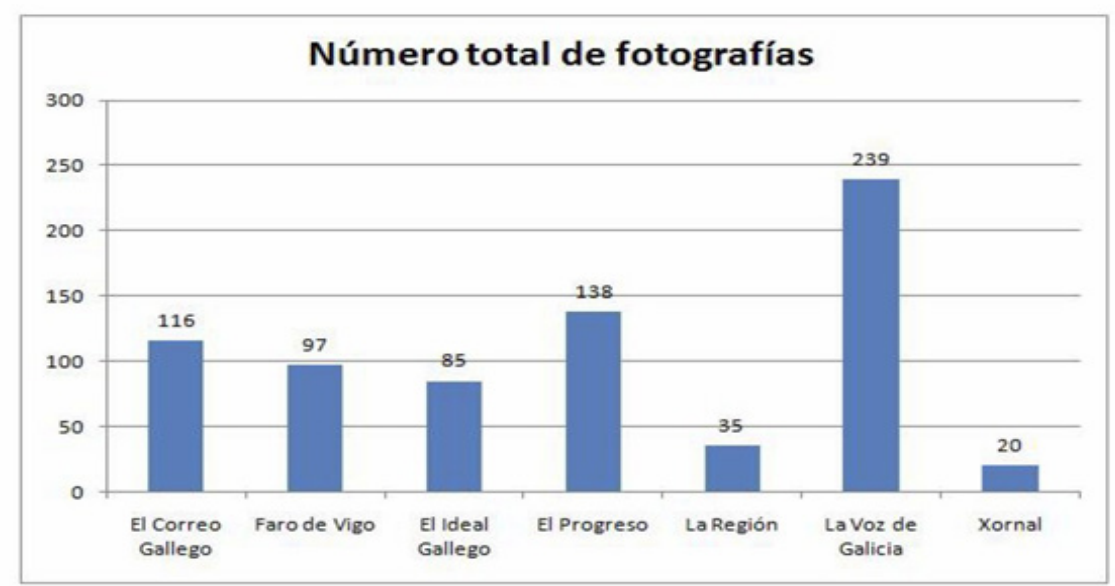

Número total de fotografias. Fuente: elaboración propia.

Entre los elementos hipermedia, en el año 2009 destacaban las galerías de imágenes, seguidas por la presencia de archivos de vídeo. En el año 2010 los archivos de sonido cobran más importancia. Se producen, por tanto, pequeños ajustes en el empleo de recursos propios de la red, aunque con un crecimiento tímido. ya que apenas hay infografías y otros elementos visuales dinámicos (lavoz. es es el cibermedio que mejores registros ofrece en todos los apartados analizados). $Y$ hay debilidades considerables: uno de los cibermedios, elidealgallego. es, no disponía de buscador en ese momento y laregion.es no aportaba datos de contacto en la portada.

El análisis de los cibermedios muestra, por tanto, un panorama de claroscuros donde destaca la apuesta decidida por tener presencia en la red y, al mismo tiempo, los escasos pasos que se dan para mejorar los productos e innovar. Para la mayoría de las empresas editoras de diarios locales de información general de pago, de momento es suficiente con estar, porque el modelo de negocio no está muy definido -apenas hay retornos económicos-, los riesgos son muchos -constatan canibalización del producto en papel- y la situación económica -la crisis afecta a los medios en papel pero también a los medios online- no permite grandes esfuerzos ${ }^{18}$. De momento se conforman con que sus productos sobrevi-

\footnotetext{
${ }^{18}$ Las entrevistas en profundidad a los responsables de los cibermedios de proximidad mostraron una gran coincidencia a la hora de analizar el panorama actual y los pasos a seguir. Los entrevistados admitieron que sus proyectos avanzan lentamente porque hay muchos interrogantes en el horizonte y tienen muchas dudas sobre el camino a seguir.
} 
van y esperan tiempos mejores.

\section{A MODO DE CONCLUSIÓN}

La industria gallega de los medios impresos y online de información general no vive ajena a las grandes transformaciones que se están produciendo en el ámbito mundial, pero tampoco se encuentra con muchas fuerzas para explorar nuevos territorios comunicativos. Desde la última década del siglo $\mathrm{XX}$, esta industria experimentó un importante desarrollo que se mantuvo hasta el año 2008, cuando se encendieron las alarmas de la recesión económica. La crisis provocó un descenso de la publicidad -superior al veinte por cien- y dibujó una nueva encrucijada a partir del año 2009, para la que todavía no existían alternativas a final del año 2010 -las estrategias de todos los cibermedios analizados consiste en posponer nuevos proyectos ambiciosos hasta que se produzca un cambio de tendencia en el mercado publicitario-.

El análisis global de los cibermedios gallegos de proximidad que promueven las empresas editoras de diarios en papel de información general permite concluir que, en líneas generales, estas iniciativas avanzan poco a poco en su intento de abandonar las pautas tradicionales del tratamiento de la información. El aprovechamiento de las posibilidades que ofrece la red es muy limitado, ya que mantienen una importante tendencia al trasvase de la información del papel a la pantalla, en lugar de elaborar productos pensados para la red. De hecho, el análisis de su contenido advierte una escasa incorporación de servicios de valor añadido a las noticias -no hay iniciativas informativas de seguimiento especial para los principales acontecimientos que se producen en la proximidad ni propuesta para implicar a las comunidades locales en los debates-. Los elementos más habituales que han incorporado es la opción de enviar las noticias por correo electrónico y la posibilidad de imprimir contenidos, pero en muy pocos casos se permite visualizar la información en PDF o disponer del recurso de la hemeroteca.

Es cierto que, aunque todos los cibermedios han ralentizado sus planes de innovación -los departamentos de nuevos proyectos apenas cuentan con personal y no cuentan con planes con cronogramas para la puesta en marcha de sus modelos que superan la fase experimental- y de desarrollo de nuevas aplicaciones -sólo introducen alguna medida para nuevas plataformas y para herramientas de reciente aparición y que han cosechado éxito-, éstos mantienen la incorporación periódica de pequeños ajustes en los productos que mejoran su calidad -realmente se trata de rediseños del producto y de aplicaciones para nuevos dispositivos-. La comparación entre los datos de 2009 y de 2010 muestra una mejora en la presentación visual -más vídeo y más multimedia- y en la organización de las vías de participación -en especial en cuestiones puntuales relevantes- para asegurar la intervención de los ciudadanos y garantizar la calidad del producto informativo final. 
Cibermedios locales: en la encrucijada por la indefinición de modelos para tiempos convulsos

\section{REFERENCIAS BIBLIOGRÁFICAS}

ARMENTIA, J.I.; CAMINOS, J.Mª; ELEXGARAY, J.; MARÍN, F. y MERCHÁN, I. (2000): El diario digital. Análisis de los contenidos textuales, aspectos formales y publicitarios. Barcelona: Bosch.

BORRAT, H. (1997): El periódico como actor político. Barcelona: Gustavo Gili.

BRUNS, A. (2005): Gatewatching: Collaborative online news production. New York: Peter Lang.

CALVO, D.; LÓPEZ, X.; y OTERO, M. (2009): Os cibermedios galegos no ano 2008. Estudo das súas características e do seu impacto no contexto comunicacional da fase actual de tránsito á sociedade da información e o coñecemento. Santiago de Compostela: Edicións Lea.

DÍAZ NOSTY, B. (coordinador) (2007): Medios de comunicación. El escenario Iberoamericano. Madrid: Ariel/Fundación Telefónica.

LÓPEZ, X.; CAMPOS, F. (2007): Os medios impresos: tempos de redefinición de estratexias. En: LÓPEZ, X. (coordinador) (2007): A Comunicación en Galicia.2007. Santiago de Compostela: Consello da Cultura Galega, pp- 16-24.

PATERSON, C.; DOMINGO, D. (2008): Making online news: The etnography of new media production. New York: Peter Lang.

REIG, R. (2007): El periodista en la telaraña. Nueva economía, comunicación, periodismo, públicos. Barcelona: Anthropos Editorial.

SALAVERRÍA, R.; GARCÍA AVILÉS, J.A.: MASIP, P.: Concepto de convergencia periodística. En: LÓPEZ, X.; PEREIRA, J. (coordinadores) (2010): Convergencia digital. Reconfiguación de los medios de comunicación en España. Santiago de Compostela: Universidad de Santiago, pp- 41-64.

VIDAL BENEYTO, J. (2002): La ventana global. Madrid: Taurus.

\section{Breve semblanza del autor}

Xosé López García es doctor en Historia y catedrático de Periodismo de la Facultad de Ciencias de la Comunicación de la Universidad de Santiago de Compostela (USC). Desde 1996 dirige el Grupo de Investigación Novos Medios (www.novosmedios.org), que desarrolla una amplia actividad centrada en el análisis de la información y la comunicación en los ámbitos local y global y en el estudio continuo de los medios de comunicación impresos y digitales.

(Recibido el 10-05-2011; aceptado el 09-02-2012) 